\title{
A Note on Parameter Identification of the AISI 304 Stainless Steel Using Micromechanical-Based Phenomenological Approaches
}

\author{
Miguel Vaz Jr. ${ }^{a *}$, Emilio Rodrigues Hulse ${ }^{a}$, Masahiro Tomiyama ${ }^{a}$ \\ ${ }^{a}$ Departamento de Engenharia Mecânica, Universidade do Estado de Santa Catarina, Joinville, SC, Brasil
}

Received: March 08, 2019; Revised: April 30, 2019; Accepted: June 10, 2019

\begin{abstract}
Austenitic stainless steels have largely been used in industrial equipment, architectural components and consumer items amongst others. Numerical simulation of manufacturing processes of such components and parts requires adequate hardening descriptions and accurate inelastic parameters. This work addresses these issues for the AISI 304 stainless steels based on phenomenological approaches using a higher order logarithmic yield stress equation and alternative micromechanical-based equations. Identification of the inelastic parameters is performed by either curve-fitting strategies or optimization techniques. The experimental-numerical comparative assessments demonstrate that the micromechanical-based yield stress equation derived from Bergström's dislocation model provides the best hardening description.
\end{abstract}

Keywords: AISI 304 stainless steel, parameter identification, inverse problems.

\section{Introduction}

Austenitic stainless steels, in especial the AISI 304 grade, have been used in a wide range of applications, such as industrial equipment (e.g. food, pharmaceutical and mining equipment), architectural components (decorative panels, ornamental work, etc.) and consumer items (sinks, cutlery, pans, etc.) amongst others. The reason lies on its good corrosion/ oxidation resistance, weldability, formability, toughness and ductility. The high industrial interest has prompted the present discussion on (i) the suitability of some selected heuristic and micromechanic-based phenomenological equations to model hardening evolution of the AISI 304 stainless steel and (ii) strategies to determine the corresponding parameters.

A successful numerical simulation of metal forming operations to manufacture AISI 304 components requires appropriate inelastic modelling. The key aspects to be observed are the hardening law and corresponding inelastic parameters, and the level of plastic deformation. In general, inelastic parameters can be obtained by using either $(a)$ curve-fitting strategies or $(b)$ inverse problem techniques. The former directly correlates true stresses and true strains under uniform deformation, whereas the latter obtains the inelastic parameter by using optimization methods associated with global measures (e.g. geometry and load information).

The literature shows that hardening parameters of stainless steels are mostly determined by curve-fitting strategies associated with tensile tests. For instance, Kashyap et al. ${ }^{1,2}$ described the hardening behaviour of the AISI 316L stainless steel under high temperature based on two and three stages of the Holloman ${ }^{3}$ relation, $\sigma=K \varepsilon^{n}$, where $\sigma$ and $\varepsilon$ are the uniaxial stresses and strains, $K$ is the strength coefficient, and $n$ is the strain-hardening exponent.
Samuel and co-workers ${ }^{4,5}$ addressed the suitability of selected hardening equations to describe plastic deformation of the AISI 316L stainless steel. The authors concluded that, in most cases, Ludwigson ${ }^{6}$ equation, $\sigma=K \varepsilon^{n}+\exp \left(K_{1}+n_{1} \varepsilon\right)$, better describes the hardening behaviour, in which $K_{1}$ and $n_{1}$ are additional inelastic constants. Similar conclusion was reached by Isaac Samuel and Choudhary ${ }^{7}$ addressing the type $316 \mathrm{LN}$ stainless steel. Following the works of Kashyap et al. ${ }^{1,2}$, Hertelé et al. ${ }^{8}$ indicate that the DIN 1.4462 duplex stainless steel can be described by two hardening stages based on the Ramberg-Osgood equation ${ }^{9}, \varepsilon=\sigma / E+K(\sigma / E)^{n}$, where $E$ is the Young's modulus. El-Magd ${ }^{10}$ investigated application of non-logarithmic yield stress equations to austenitic steels. The author adopted Voce's ${ }^{11}, \sigma=a+b[1-\exp (-c \varepsilon)]$, and a modified version of Voce's yield stress equation, $\sigma=C_{1}+$ $C_{2} \varepsilon+C_{3}\left[1-\exp \left(-C_{4} \varepsilon\right)\right]$, to describe plastic deformation of the austenitic steels X6CrNi18-11 and X8CrNiMoNb16-16, respectively, where $a, b, c, C_{1}, C_{2}, C_{3}$ and $C_{4}$ are material parameters. Hardening descriptions for the AISI 316LN were discussed by Ashraf et al. ${ }^{12}$ for cyclic loading, who also adopted Voce's ${ }^{11}$ yield stress equation to model isotropic hardening.

Contrasting to curve-fitting strategies, application of optimization-based parameter identification techniques to austenitic stainless steels has been rarely reported in the literature. Notwithstanding, preliminary issues on use of optimization techniques to obtain inelastic parameters of the AISI 304 were discussed by the authors in Vaz Jr. et al. ${ }^{13}$. However, it is important to mention that use of optimization methods is well established and has been used to determine inelastic parameters for both metallic and non-metallic materials. For instance, the authors addressed use of different optimization methods to parameter identification of materials as different as AISI 1045 carbon steel and high-density polyethylene (HDPE) ${ }^{14}$. 
The present work aims to further discuss hardening descriptions of the AISI 304 stainless steels based on phenomenological approaches using $(A)$ a higher order logarithmic yield stress equation and $(B)$ alternative micromechanical-based equations. The corresponding parameters are determined by using either curve-fitting techniques (logarithmic equation) or optimization methods (micromechanical-based equations). The article is organized as follows: section 2 presents a third-order logarithmic yield stress equation and two hardening equations based on micromechanical concepts. Section 3 introduces parameter identification based on optimization strategies using a composed objective function. Application of the aforementioned hardening equations and parameter identification methods is presented in section 4 . The main conclusions and final remarks are presented in section 5 .

\section{Micromechanical-based and phenomenological yield stress equations}

The brief review shows that stainless steels present a peculiar hardening behaviour, frequently described by models based on multiple hardening stages or non-logarithmic equations. Furthermore, the AISI 304 stainless steel is subject to deformation-induced phase transformation of austenite to martensite, causing inflections in the stress-strain curve. However, De et al. ${ }^{15}$ show for the AISI 304 steel that, for low strain rates and temperatures higher than $298 \mathrm{~K}$ (as defined in the present experiments), such effects do not take place, making possible to model inelastic deformation using straightforward phenomenological approaches.

\subsection{Heuristic phenomenological yield stress equations}

The literature shows a wide range of empirical stressstrain relations, such as the classical equations proposed by Hollomon $^{3}$, Ludwigson ${ }^{6}$ and Swift ${ }^{16}$. The material parameters of most such equations are determined by curve-fitting procedures of true stresses and true strains in a logarithmic plot. This technique requires the assumption of uniform deformation, thereby restricting evaluation of strains and stresses up to necking onset (instability). The literature review presented in section 1 highlights the nonlinear dependence of logarithmic stresses on logarithmic strains exhibited by the AISI 304 stainless steel (oftentimes modelled by two ${ }^{1,7}>$ or three ${ }^{2}$ hardening stages). Therefore, the present work investigates the suitability of a higher order logarithmic yield stress curve.

Within this framework, Dimatteo et al. ${ }^{17}$ proposed a $3^{\text {rd }}$ order $(\ln \sigma) \times(\ln \varepsilon)$ logarithmic fit to Dual Phase (DP) $450 / 600 / 800 / 1000$ and Transformation Induced Plasticity (TRIP) 800 steels attempting to predict the influence of alloying elements and heat treatment conditions in a simple manner.
The present work investigates a possible extension to the AISI 304 stainless steel. Complementing the equation introduced by Dimatteo et al. ${ }^{17}$, this study includes a provision for elastic deformations, so that

$$
\begin{aligned}
\ln \left(\sigma_{Y}\right) & =A\left[\ln \left(\bar{\varepsilon}_{p}+\varepsilon_{0}\right)\right]^{3}+B\left[\ln \left(\bar{\varepsilon}_{p}+\varepsilon_{0}\right)\right]^{2} \\
& +C\left[\ln \left(\bar{\varepsilon}_{p}+\varepsilon_{0}\right)\right]+D,
\end{aligned}
$$

where $A, B, C$, and $D$ are the model parameters to be determined by the curve-fitting procedure, $\varepsilon_{0}$, is the initial strain which is obtained by solving the cubic-logarithmic equation resulted by the intersection between the elastic and plastic curves, $A\left[\ln \left(\varepsilon_{0}\right)\right]^{3}+B\left[\ln \left(\varepsilon_{0}\right)\right]^{2}+(C-1)\left[\ln \left(\varepsilon_{0}\right)\right]+$ $(D-\ln E)=0$, in which $E$ is the Young's modulus.

\subsection{Micromechanical-based yield stress equations}

Phenomenological yield stress models can be approximated from micromechanical-based concepts. This section summarises strategies to obtain two such equations. The approach accounts for physical mechanisms of dislocation theory assuming a Taylor-like correlation ${ }^{18}$ between the yield stress, $\sigma_{Y}$, and total dislocation density, $\rho$, as

$$
\sigma_{Y}=\sigma^{*}+M \alpha G b\left(\sqrt{\rho}-\sqrt{\rho^{*}}\right),
$$

where $M$ is a factor which correlates the shear flow stress of a single crystal and the uniaxial flow stress of a polycrystal, $\alpha$ is a parameter which depends on the dislocation/dislocation interaction and thermal activation kinetics, $G$ is the shear modulus, $b$ is the magnitude of the Burgers vector, and $\sigma^{*}$ and $\rho *$ are reference values. All micromechanical parameters are assumed constant in the present approximation. The following paragraphs summarizes key aspects of two alternative phenomenological yield stress equations obtained from Eq. (2).

Modified Voce's yield stress equation: Voce's original equation was proposed based on empirical considerations ${ }^{11}$. However, El-Magd ${ }^{10}$ demonstrated that this hardening law can also be derived from micromechanical approach. Nevertheless, the author indicated that its application to austenitic steel X6CrNi18-11 at different temperatures provided good approximation only in the range of relatively small plastic strains up to $0.2^{10}$.

Following similar considerations, based on Mecking and Kocks'19 evolution of the dislocation density, $d \rho / d \varepsilon_{p}=k_{1}^{M K} \sqrt{\rho}+k_{2}^{M K} \rho$, a better representation for larger plastic strains can be derived by assuming a constant dynamic recovery rate, $k_{2}^{M K}$, and by approximating the effects of the dislocation density and dislocation free path, $l$, upon the dislocation storage rate ${ }^{10}$ as $k_{1}^{M K}=1 /(l \sqrt{\rho})=k^{*}\left(1+c^{*} \varepsilon_{\rho}\right)$,where $k^{*}$ and $c^{*}$ are constants of the model. The evolution of the dislocation density is, therefore, $d \rho / d \varepsilon_{p}=k^{*}\left(1+c^{*} \varepsilon_{p}\right) \sqrt{\rho}+k_{2}^{M K} \rho$. 
The yield stress equation is determined by solving the differential equation, $d \sigma_{Y} / d \varepsilon_{p}=\left(d \sigma_{Y} / d \rho\right)\left(d \rho / d \varepsilon_{p}\right)$, where $d \sigma_{Y} / d \rho$ is obtained from Eq. (2), so that

$$
\sigma_{Y}=\sigma_{0}^{t}+s^{t} \bar{\varepsilon}_{p}+\left(\sigma_{\infty}^{t}-\sigma_{0}^{t}\right)\left[1-\exp \left(-\delta^{t} \bar{\varepsilon}_{p}\right)\right]
$$

in which $\sigma_{0}^{t}$ and $\sigma_{\infty}^{t}$ are the initial stress and saturation stress, respectively, $\varsigma^{t}$ is the linear hardening coefficient, and $\delta^{t}$ is the rate of approximation to the linear hardening. The correspondence between the phenomenological and micromechanical parameters obtained from solution of the differential equation is presented in Table 1. Phenomenological hardening laws similar to Eq. (3) have been referred as Modified Voce model. The literature shows increasing use of this hardening equation to describe the yield stress evolution of different materials, such as the DP 780 / TRIP 780 high strength steel ${ }^{20}$ and $\mathrm{X} 8 \mathrm{CrNiMoNb16-16}$ austenitic steel ${ }^{10}$.

Bergström's yield stress equation: Bergström ${ }^{21}$ proposed a micromechanical stress-strain relation for polycrystalline monophasic steels based on the behaviour of large number of mobile and immobile dislocations. In this case, the evolution of the dislocation density with respect to plastic deformation was derived as $d \rho / d \varepsilon_{p}=U^{B}-A^{B}-\Omega^{B} \rho$, where $\Omega^{\mathrm{B}}$ is associated with mobilization and annihilation of dislocations, $A^{B}$ and $U_{B}$ represent parameters which account for mobile dislocations and its corresponding rate of increase, respectively. The phenomenological yield stress equation is derived from $d \rho / d \varepsilon_{p}$ and Eq. (2) as

$$
\begin{gathered}
\sigma_{Y}=\sigma_{0}^{b}-\sigma_{k}^{b}+\left\{\left(\sigma_{\infty}^{b}-\sigma_{0}^{b}+\sigma_{k}^{b}\right)^{2} \mid\right. \\
\left.\left[1-\exp \left(-\delta^{b} \bar{\varepsilon}_{p}\right)\right]+\sigma_{k}^{b^{2}} \exp \left(-\delta^{b} \bar{\varepsilon}_{p}\right)\right\}^{1 / 2},
\end{gathered}
$$

where $\sigma_{0}^{b}$ is the initial stress, $\sigma_{\infty}^{b}$ is the saturation stress, $\sigma_{k}^{b}$ is the stress correction for the initial dislocation density, $\rho_{0}$, and $\delta^{\mathrm{b}}$, is the rate to approximate the saturation stress.
Table 1 also presents the correspondence between phenomenological and micromechanical parameters for Bergström's hardening equation obtained from solution of the differential equation

\section{Identification of the material parameters}

Material parameters of yield stress equations can be determined either by curve-fitting strategies or by inverse problem techniques. The former obtains the inelastic parameters directly from fitted true stress - true strain curves, whereas the latter uses optimization methods to approximate computed and measured global/local quantities.

Curve-fitting strategies: The present work makes use of tensile tests to obtain experimental data to determine the inelastic parameters. Tensile tests of ductile materials involve five general stages/events: (i) elastic deformation; (ii) plastic deformation under uniform stress states; (iii) necking onset; (iv) inelastic deformation under triaxial stress states; and (v) catastrophic/macroscopic failure. The technique fits the yield stress equation in a true stress/true strain logarithmic plot. However, curve fitting must be performed under uniaxial conditions and, therefore, comprises stage (ii) up to necking onset (i.e. instability) in order to avoid triaxial stress/strain states.

Inverse problem techniques: The inverse problem techniques use optimization methods to determine material parameters of the yield stress equations. The method minimizes the differences between experimental measures and corresponding computed responses with respect to a given norm ${ }^{22}$. In order to better capture the effects of plastic deformations and improve assessment of the parameters obtained by the optimization method, this work adopts evolution of both tensile load, $R_{L}$, and specimen central radius, $R_{D}$, measured at selected deformation stages. In the present work no uncertainties were accounted for.

Table 1. Phenomenological correspondence of micromechanical parameters.

\begin{tabular}{cc}
\hline Equation & Hardening parameters \\
\hline Modified Voce & $\sigma_{0}^{t}=\sigma i$ \\
$\sigma_{\infty}^{t}=\sigma^{*}-M \alpha G b\left[\sqrt{\rho^{*}}-\left(1+2 c^{*} / k_{2}^{M K}\right)\left(k^{*} / k_{2}^{M K}\right)\right]$ \\
$S^{t}=M \alpha G b c^{*}\left(k^{*} / k_{2}^{M K}\right)$ \\
$\delta^{t}=k_{2}^{M K} / 2$ \\
$\sigma_{0}^{b}=\sigma^{*}-M \alpha G b\left(\sqrt{p^{*}}-\sqrt{p_{0}}\right)$ \\
$\sigma_{\infty}^{b}=\sigma^{*}-M \alpha G b\left[\sqrt{\rho^{*}}-\left(U^{B}-A^{B}\right)^{2} / \Omega^{B^{2}}\right]$ \\
$\sigma_{k}^{b}=M \alpha G b \sqrt{\rho_{0}}$ \\
$\delta^{b}=\Omega^{B}$
\end{tabular}


Unconstrained nonlinear optimization is used in the present parameter identification problem, which can be generally stated as

$$
\begin{array}{lll}
\text { Minimise } & g(p)=\lambda_{L} g_{L}(p)+\lambda_{D} g_{D}(p) & \boldsymbol{p} \in R^{n_{p}} \\
\text { Such that } & p_{t}^{\text {inf }} \leq p_{i} \leq p_{i}^{\text {sup }} & i=1, \cdots, n_{p}
\end{array}
$$

where

$$
\begin{aligned}
& g_{L}(\boldsymbol{p})=\sqrt{\frac{1}{N_{L}} \sum_{j=1}^{N_{L}}\left[\frac{R_{L, j}^{E x p}-R(\boldsymbol{p})_{L, j}^{N u m}}{R_{L, j}^{E x p}}\right]^{2}} \text { and } \\
& g_{D}(\boldsymbol{p})=\sqrt{\frac{1}{N_{D}} \sum_{j=1}^{N_{D}}\left[\frac{R_{D, j}^{E x p}-R(\boldsymbol{p})_{D, j}^{N u m}}{R_{D, j}^{E x p}}\right]^{2}}
\end{aligned}
$$

are individual objective functions for the load (subscript $L$ ) and specimen central radius (subscript $D$ ), $N_{L}$ and $N_{D}$ are corresponding number of experimental points, $\boldsymbol{p}=\left[p_{1} p_{2} \cdots p_{i} \cdots p_{n_{p}}\right]^{T}$ is the design vector containing $n_{p}$ design variables $p_{i}$ (material parameters), and $p_{i}^{\text {inf }}$ and $p_{i}^{\text {sup }}$ are minimum and maximum values of each material parameter. The weights $\lambda_{L}$ and $\lambda_{D}$ are the contribution of each measure to the global objective function which, in the present study, were assumed $0 \leq \lambda_{L}$, $\lambda_{D} \leq 1$ and $\lambda_{L}+\lambda_{D}=1$.

The optimization technique adopted in this work uses the gradient-free downhill simplex method, also known as Nelder-Mead (NM) algorithm ${ }^{23}$. It is important to mention that, in principle, any optimization method can be used in conjunction with the proposed strategy. The NM algorithm was selected owing to its high success rate and computational cost-wise competitiveness in similar problems when compared against heuristic and gradient-based schemes ${ }^{14}$. The method defines a regular polytope of $n_{p}+1$ vertices for a $n_{p}$ number of material parameters, which contracts or moves towards the minimum by replacing the worst vertex. Selection of the new vertex is performed after the following possible operations: reflection, expansion, internal/external contraction or shrinkage. Convergence is assumed when the normalized global fitness with respect to the best and worst vertices reaches a pre-defined value. The reader is referred to Nelder and $\mathrm{Mead}^{23}$ and Lagarias et al. ${ }^{24}$ for additional details on the NM optimization scheme, and Vaz Jr. et al. ${ }^{25}$ for the implementation used in this work.

\section{Results and Discussions}

This section discusses parameter identification of the AISI 304 stainless steel using curve fitting and optimization methods.
Tensile tests of cylindrical specimens prepared according to the Brazilian ABNT NBR ISO $6892^{26}$ were adopted to determine the inelastic parameters of Eq. (1) and Eqs. (3)(4). The chemical composition of the austenitic AISI 304 steel used in this study is presented in Table 2.

The specimens were manufactured with a gauge section diameter $d_{0}=10 \mathrm{~mm}$, initial gauge length $l_{0}=50 \mathrm{~mm}$ (measurements performed by a clip gauge transducer), total gauge section $L=l_{0}+2 d_{0}=70 \mathrm{~mm}$, shoulder diameter $d_{s}$ $=20 \mathrm{~mm}$ and a transitional radius $r_{t}=15 \mathrm{~mm}$ between the gauge section and shoulder. The tests were performed under displacement control with maximum crosshead speed $v_{c}=3 \mathrm{~mm} /$ $\min$ (low strain rate). Evolution of the specimen central radius is estimated by assuming plastic deformation at constant volume up to necking onset, so that $R_{D}=R_{0} /(\mathrm{e}+1)^{1 / 2}$, where $R_{0}$ is the initial central radius and $e=\Delta l / l_{0}$ is the specimen elongation (engineering strain). The reference radius data are complemented by measurement of the final specimsen central radius.

The geometrical model used in the simulations consider axisymmetry around the rotation axis $Z-Z^{\prime}$ and symmetry about the $R-R^{\prime}$ axis, making possible to model only $1 / 4$ of the specimen. A structured, eight-noded quadrilateral finite element mesh with 200 elements and 661 nodes with progressive refinement towards the specimen $R-R^{\prime}$ axis was used.

\subsection{Curve-fitting of the third-order logarithmic yield stress equations}

The curve-fitting strategy accounts for load evolution from plastic onset up to necking. The elastic limit is the lower bound imposed by the elastic curve, whereas the maximum load is assumed as the instability limit (necking onset). The true strains and true stresses are determined as $\varepsilon=\ln (1$ $+e)$ and $\sigma=s(1+e)$, respectively, where $s=R_{L} / A_{0}$ is the engineering stress, $R_{L}$ is the axial load, and $A_{0}$ is the initial cross-sectional area. The reader is referred to Davis ${ }^{27}$ for a detailed description of the curve-fitting method, including experimental considerations and requirements.

Figure 1(a) shows the experimental data and fitted equation in a $(\ln \sigma) \times(\ln \varepsilon)$ plot, whereas Table 3 presents the corresponding material parameters. For the sake of clarity, not all experimental points are represented in Figure 1(a) and forthcoming figures. The experimental data show that the yield stress of the AISI 304 stainless steel at room temperature prompts a nonlinear correlation between stresses and strains in the $(\ln \sigma) \times(\ln \varepsilon)$ space, thereby precluding using of first-order logarithmic equations such as Hollomon's ${ }^{3}$ and Swift's ${ }^{16}$. On the other hand, the $3^{\text {rd }}$ order logarithmic curve achieved an excellent fit with a coefficient of determination $R^{2}=0.999187$.

Table 2. Chemical composition of the AISI 304 steel (in wt.\%)(*).

\begin{tabular}{lcccccc}
\hline & $\mathrm{Cr}$ & $\mathrm{Ni}$ & $\mathrm{C}$ & $\mathrm{Mn}$ & $\mathrm{P}$ & $\mathrm{S}$ \\
\hline Nominal & $18-20$ & $8-10.5$ & $\max 0.08$ & $\max 2.0$ & $\max 0.045$ & $\max 0.03$ \\
This work & 18.677 & 8.780 & 0.07612 & 1.9856 & 0.01984 & 0.02767 \\
\hline
\end{tabular}

${ }^{(*)}$ Mass spectrometer - Shimadzu OES 5500II 
Table 3. Curve-fitting parameters for the third-order $(\ln ) \mathrm{x}(\ln \varepsilon)$ logarithmic curve.

\begin{tabular}{lcc}
\hline & Symbol & Value \\
\hline & $\mathrm{A}$ & $3.54744 \times 10^{-3}$ \\
& $\mathrm{~B}$ & $8.12467 \times 10^{-3}$ \\
$3^{\text {rd }}$ order polynomial equation & $\mathrm{C}$ & 0.607523 \\
& $\mathrm{D}$ & 21.3091 \\
Coefficient of determination & $\varepsilon_{0}$ & $1.97659 \times 10^{-3}$ \\
\hline
\end{tabular}

(a) Logarithmic fitting.

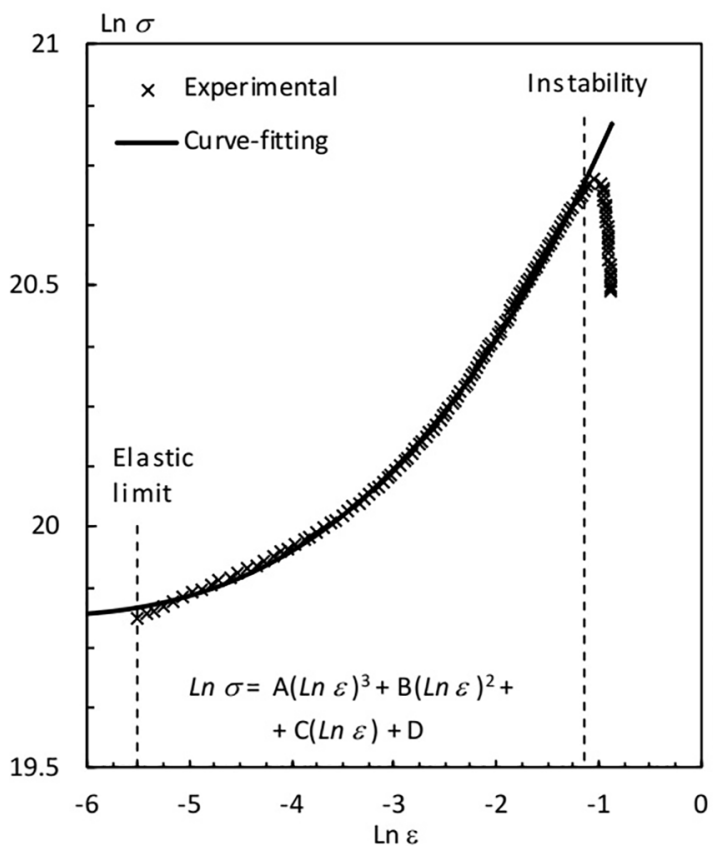

(b) Load and radius evolution.

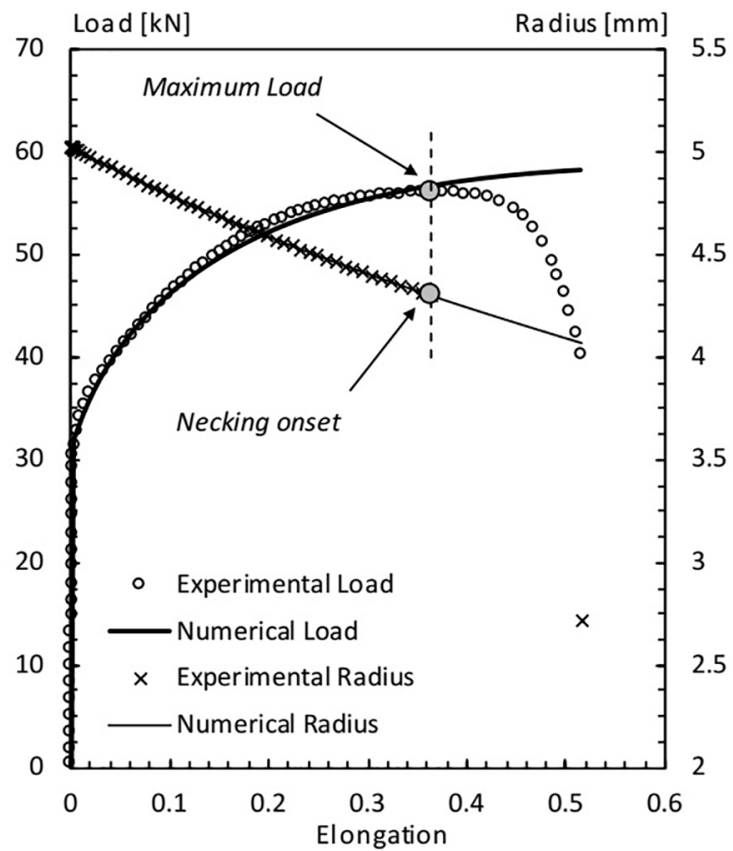

Figure 1. Third-order $(\ln \sigma) \times(\ln \varepsilon)$ logarithmic curve: (a) Fitting of the third-order equation and (b) evolution of the tensile load and specimen central radius.

Verification of the $3^{\text {rd }}$ order logarithmic equation is performed by comparing load and central radius computed by finite elements (using the aforementioned geometrical model) against available reference data. In this case, the yield stress is defined by Eq. (1) with hardening parameters given in Table 3. Figure 1(b) indicates a very accurate prediction of tensile loads and specimen central radius up to necking onset. However, the simulations show that both tensile load and specimen central radius are grossly overestimated after the maximum experimental load. In this case, no necking was observed, causing the computed load to further increase towards the final stages and macroscopic failure. Therefore, use of the $3^{\text {rd }}$ order logarithmic equation is restricted to relatively small plastic strains, represented by the equivalent plastic strain limit $\bar{\varepsilon}_{p} \leq 0.317$.

\subsection{Application of the optimization-based technique to the Modified Voce and Bergström yield stress equations}

Use of optimization methods makes possible to determine inelastic parameters taking into account experimental data up to macroscopic failure. In the present study, the tensile load and specimen central radius constitute the reference measures. The global error function is represented in Eq. (5), whereas the individual objective functions are given by Eqs. (6). The global error $g(\boldsymbol{p})$ constitutes a weighted sum of the individual fitness $g_{L}(\boldsymbol{p})$ and $g_{D}(\boldsymbol{p})$ by $\lambda_{L}$ and $\lambda_{D}$. This strategy allows a straightforward investigation of the effects of each type of reference measure (load / central radius) in the final hardening parameters. In order to quantify such effects a new index $G(\boldsymbol{p}, \lambda)$ is defined as 


$$
G(\boldsymbol{p}, \lambda)=\sqrt{\frac{1}{2}\left[\left(\frac{g_{L}(\boldsymbol{p})-g_{L}^{\min }}{g_{L}^{\min }}\right)^{2}+\left(\frac{g_{D}(\boldsymbol{p})-g_{D}^{\min }}{g_{D}^{\min }}\right)^{2}\right]},
$$

where $g_{L}^{\min }\left(\lambda_{L}=1 ; \lambda_{D}=0\right)$ and $g_{D}^{\min }\left(\lambda_{L}=0 ; \lambda_{D}=1\right)$ are the best individual fitness for tensile load and central radius, respectively. Qualitatively, the index $G(\boldsymbol{p}, \lambda)$ represents the average differences between computed load and central radius for a given weight set $\left\{\lambda_{L}, \lambda_{D}\right\}$ and their best individual approximation.

Three cases were studied for both Modified Voce and Bergström yield stress equations. Case $A$ : parameter identification accounts for only tensile loads $\left(\lambda_{\mathrm{L}}=1 ; \lambda_{\mathrm{D}}=0\right)$ and provides $g_{L}^{\min }$ (the individual fitness, $g_{D}$, for the specimen central radius is calculated but not included in the computation of the global

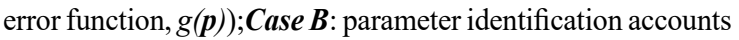
for only the specimen central radius $\left(\lambda_{L}=0 ; \lambda_{D}=1\right)$ and provides $g_{D}^{\min }$ the individual objective function, $g_{L}$, for tensile load is computed but not included in the computation of $g(\boldsymbol{p})$ ); and Case $\boldsymbol{C}$ : tensile loads and specimen central radius impose equal effect on obtaining the hardening parameters $\left(\lambda_{L}=0.5 ; \lambda_{D}=0.5\right)$. Table 4 presents the individual fitness, $g_{L}(\boldsymbol{p})$ and $g_{D}(\boldsymbol{p})$, and the global index, $G(\boldsymbol{p}, \lambda)$, for all cases. The best individual fitness, $g_{L}^{\min }$ and $g_{D}^{\min }$, are represented in boldface. The corresponding load and central radius evolution curves for Modified Voce and Bergström yield stress equations are shown in Figures 2 and 3, respectively.

It is relevant to notice from Table 4 and Figures 2 and 3 that, for both yield stress equations, parameter identification based solely on the specimen central radius (Case B:: $\lambda_{L}=0$; $\lambda_{D}=1$ ) does not guarantee acceptable approximations of the loading curve. The tensile loads are clearly underestimated for both Modified Voce (Figure 2a) and Bergström (Figure 3a) equations. It can be observed in Table 4, that the individual objective function for the load, $g_{L}$, is substantially larger

Table 4. Modified Voce and Bergström equations: best individual fitness(*) and global index.

\begin{tabular}{lcccccc}
\hline \multirow{2}{*}{ Hardening Equation } & Case & & Weights & \multicolumn{2}{c}{ Individual fitness } & \multicolumn{2}{c}{ Global index } \\
& & $\lambda_{L}$ & $\lambda_{D}$ & $g_{L}(\boldsymbol{p}) \times 10^{2}$ & $g_{D}(\boldsymbol{p}) \times 10^{2}$ & $G(\boldsymbol{p}, \lambda)$ \\
\hline \multirow{3}{*}{ Modified Voce } & $A$ & 1 & 0 & $\mathbf{0 . 7 9 4 4 6 9}$ & 0.879569 & 14.316 \\
& $B$ & 0 & 1 & 15.1765 & $\mathbf{0 . 0 4 1 3 9 9 6}$ & 12.801 \\
& $C$ & 0.5 & 0.5 & 1.18416 & 0.193070 & 2.614 \\
Bergström & $A$ & 1 & 0 & $\mathbf{0 . 7 7 5 8 7 6}$ & 0.378298 & 5.106 \\
& $B$ & 0 & 1 & 14.2041 & $\mathbf{0 . 0 4 6 0 1 7 5}$ & 12.238 \\
& $C$ & 0.5 & 0.5 & 0.882198 & 0.163666 & 1.810 \\
\hline
\end{tabular}

${ }^{\left({ }^{*}\right)}$ and $g_{L}^{\min }$ are $g_{D}^{\min }$ represented in boldface.

(a) Loading curve.

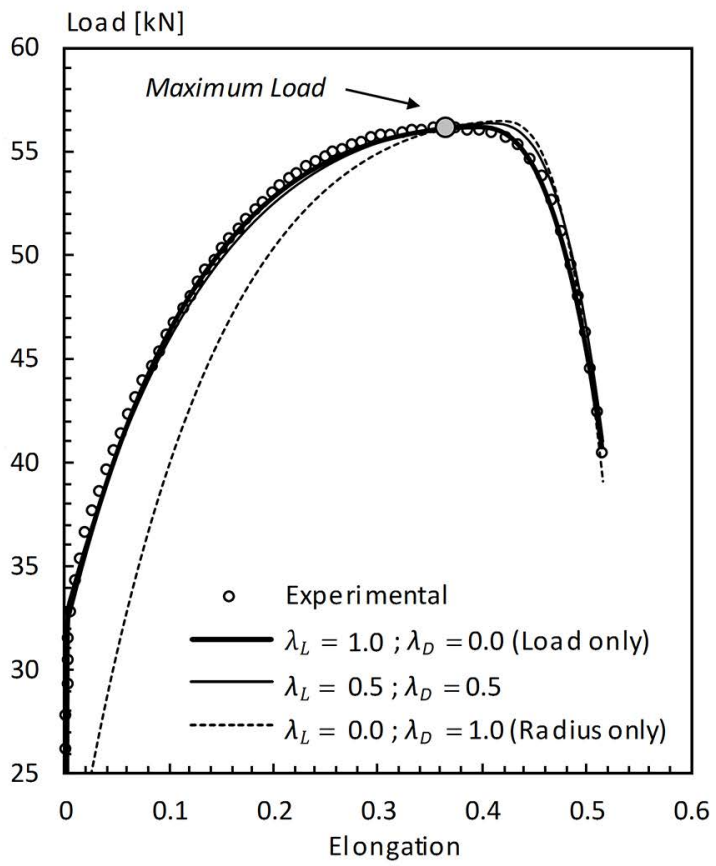

(b) Central radius.

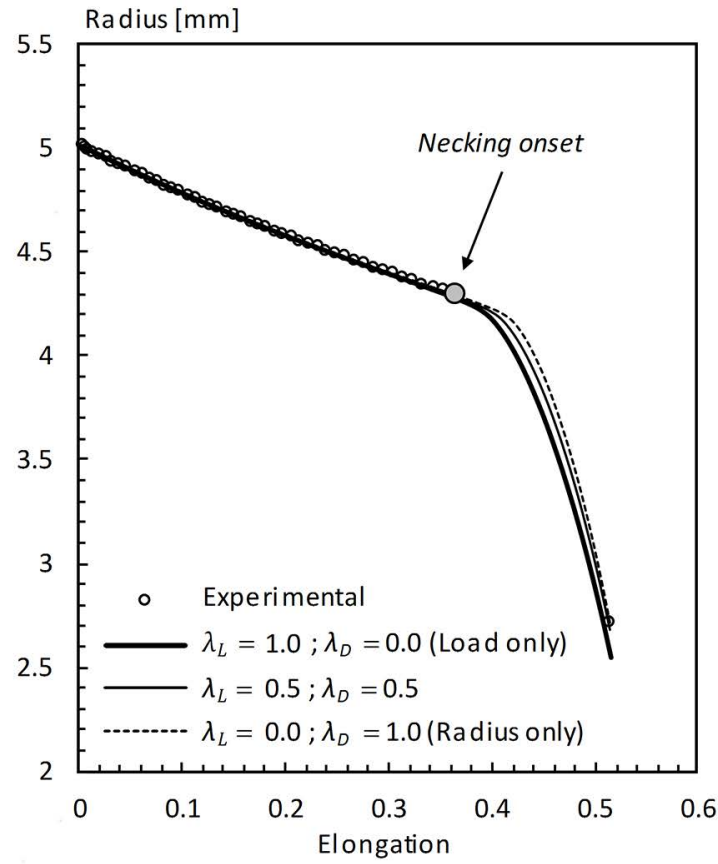

Figure 2. Modified Voce's yield stress equation: evolution of the tensile load and specimen central radius. 
(a) Loading curve.

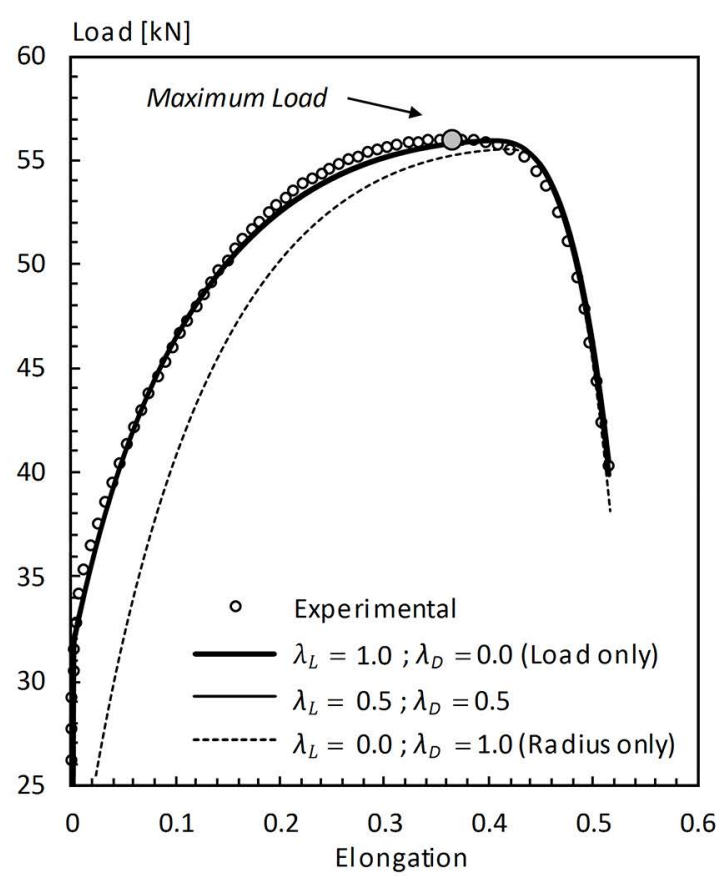

(b) Central radius.

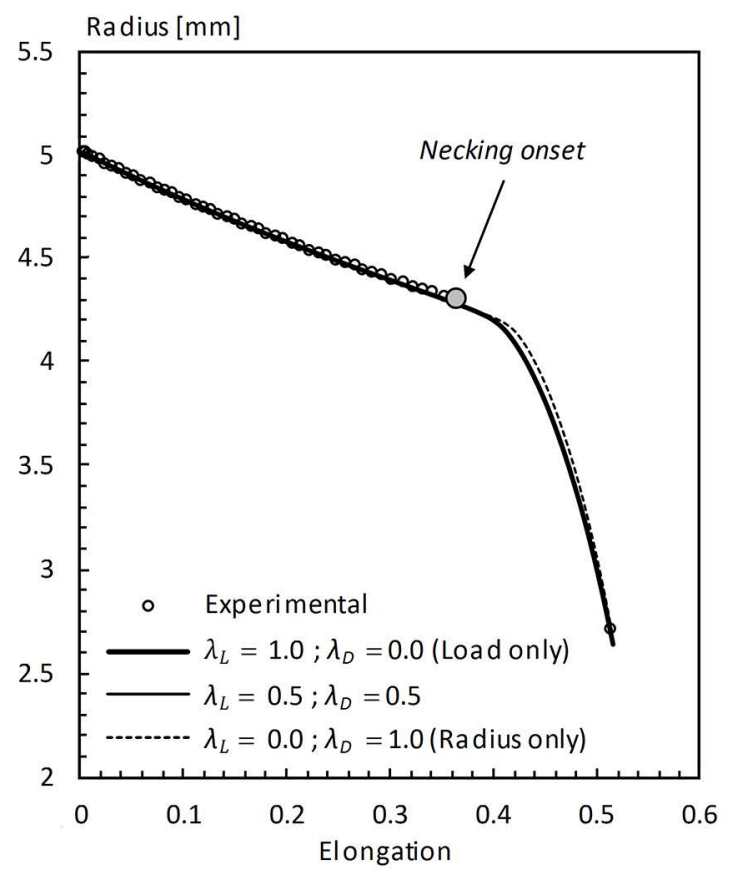

Figure 3. Bergström's yield stress equation: evolution of the tensile load and specimen central radius.

than their best minimum $g_{L}^{\min }$ for both yield equations. A similar behaviour, yet in a smaller scale, can be observed when parameter identification is performed based solely on the tensile load (Case A: $\lambda_{L}=1 ; \lambda_{D}=0$ ). In this case, the greatest effect takes place in the final specimen central radius, reaching a maximum error of $6.1 \%$ with respect to the experimental measure.

The best results are obtained when the influence of the tensile load and specimen central radius are accounted for (Case $\boldsymbol{C}: \lambda_{L}=0.5 ; \lambda_{D}=0.5$ ). Table 5 presents the material parameters for Case $\boldsymbol{C}$ for both Modified Voce and Bergström yield equations. The quantitative assessment for $\operatorname{Case} \boldsymbol{C}$ is also shown in Table 4, which indicates that the global index, $G(\boldsymbol{p}, \lambda)$, are markedly smaller than those evaluated for Case $\boldsymbol{A}$ and Case $\boldsymbol{B}$.

The good approximations obtained for Case $\boldsymbol{C}$ are illustrated in Figure 4, which shows the finite element mesh and actual specimen at final stages of deformation for Bergström and Modified Voce equations. The geometrical differences between the actual specimen and simulations are very small, virtually imperceptible in Figures 4(a) and 4(b). The equivalent plastic strain, $\bar{\varepsilon}_{p}$, for both yield stress equations are also presented in Figure 4. The differences of $\overline{\boldsymbol{\varepsilon}}_{p}$ computed using Bergström and Modified Voce equations are also very small, reaching a maximum of $0.1 \%$ at the centre of the specimen.
Table 5. Final parameters for weights $\lambda_{L}=0.5$ and $\lambda_{D}=0.5$.

\begin{tabular}{ccc}
\hline \multicolumn{1}{c}{ Equation } & Symbol & Value \\
\hline Modified Voce & $\sigma_{0}^{t}$ & $415.223[\mathrm{MPa}]$ \\
& $\sigma_{\infty}^{t}$ & $1049.68[\mathrm{MPa}]$ \\
$S^{t}$ & $383.664[\mathrm{MPa}]$ \\
$\delta^{t}$ & 3.75756 \\
$g\left(\boldsymbol{p}^{t}\right)=\lambda_{L} g_{L}\left(\boldsymbol{p}^{t}\right)+\lambda_{D} g_{D}\left(\boldsymbol{p}^{t}\right)$ & $6.88615 \times 10^{-3}$ \\
\hline & $\sigma_{0}^{b}$ & $405.110[\mathrm{MPa}]$ \\
& $\sigma_{\infty}^{b}$ & $1726.55[\mathrm{MPa}]$ \\
$\sigma_{k}^{b}$ & $453.044[\mathrm{MPa}]$ \\
$\delta^{b}$ & 1.05480 \\
$g\left(\boldsymbol{p}^{b}\right)=\lambda_{L} g_{L}\left(\boldsymbol{p}^{b}\right)+\lambda_{D} g_{D}\left(\boldsymbol{p}^{b}\right)$ & $5.22932 \times 10^{-3}$ \\
\hline
\end{tabular}

Remark: The results discussed in previous paragraphs show that identification of material parameters for both Bergström and Modified Voce equations using optimization techniques provide good results for both load evolution and geometrical configuration. Notwithstanding, Table 4 shows that, for weights $\lambda_{L}=0.5$ and $\lambda_{D}=0.5$ (Case $\boldsymbol{C}$ ), Bergström equation provides individual errors, $g_{L}$ and $g_{D}, 34.2 \%$ and $18.0 \%$ smaller for tensile loads and specimen central radius, respectively, when comparing against Modified Voce hardening law. 


\section{(a) Bergström yield stress equation}
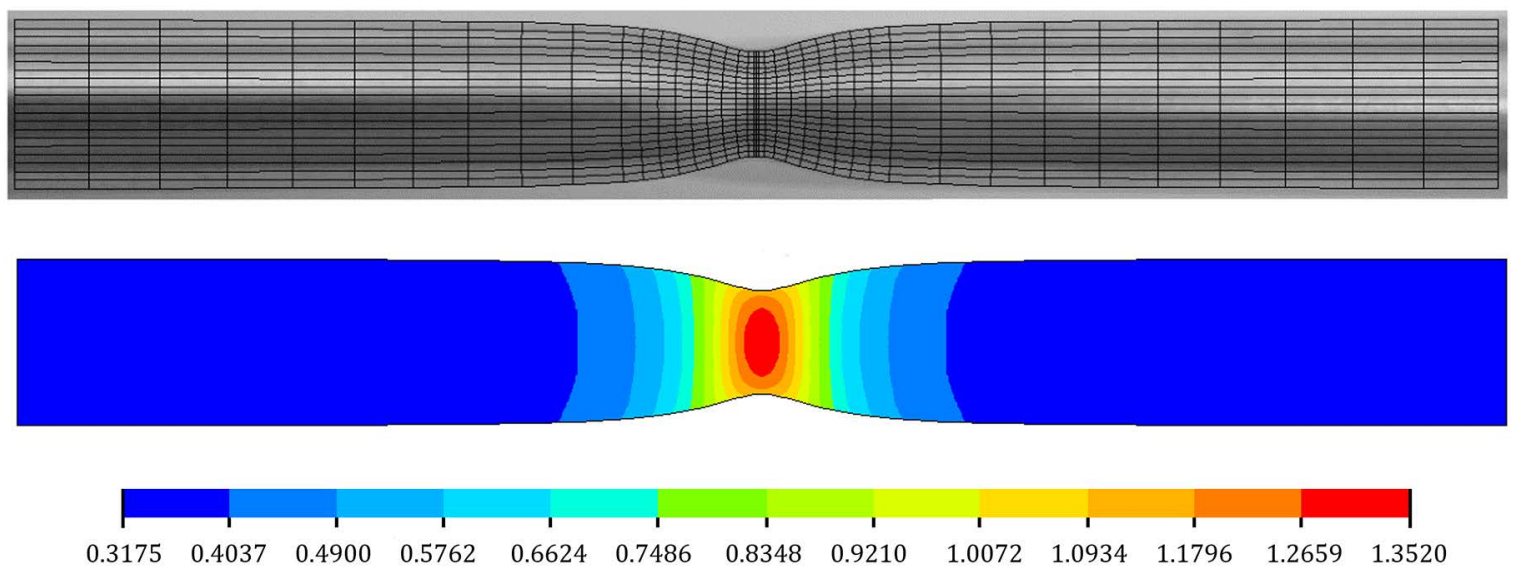

\section{(b) Modified Voce yield stress equation}
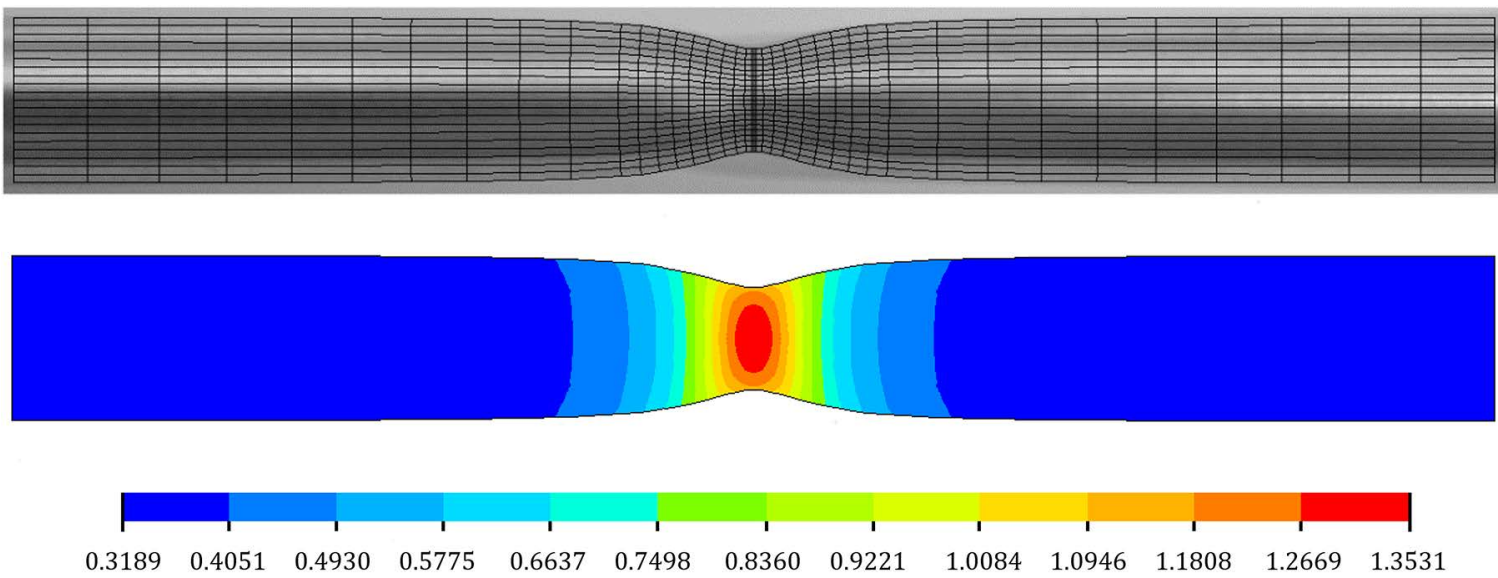

Figure 4. Finite element mesh and actual specimen at final stage of deformation and corresponding distribution of the equivalent plastic strain for (a) Bergström and (b) Modified Voce equations.

Furthermore, the global errors, $g(\boldsymbol{p})$, (see Table 5) for Bergström equation is $31.7 \%$ smaller than those evaluated by the Modified Voce equation. Therefore, based on the results discussed in the previous paragraphs, Bergström hardening law is recommended to represent the yield behaviour of the AISI 304 stainless steel.

\section{Concluding Remarks}

The AISI 304 stainless steel has been largely used in manufacturing equipment and components owing its balance between good mechanical and corrosion characteristics and relative lower cost (when compared to other stainless steels).
Therefore, a hardening description able to describe accurately inelastic deformation in a simplified manner is of great industrial interest. Many researchers agree that a purely micromechanical yield stress criterion is the best and the most elegant approach to account for strain induced phase transformation from austenite to martensite. On the other hand, the present phenomenological equations have shown able to approximate load and geometry evolution with acceptable accuracy, making possible its straightforward use in combination with commercial software. The present work is inserted within this framework and discusses use of a heuristic third-order logarithmic equation and two micromechanical-based phenomenological yield stress equations to describe hardening evolution of the AISI 304 stainless steel. 
The material parameters of the hardening equations were determined by either curve fitting (third-order logarithmic equation) or inverse problem techniques (micromechanicalbased yield stress equations).

Curve-fitting of the third order $(\ln \sigma) \times(\ln \varepsilon)$ curve indicates good results up to necking onset, corresponding to a maximum equivalent plastic strain $\overline{\boldsymbol{\varepsilon}}_{p}=0.317$. Contrastingly, the optimization-based parameter identification was able to approximate both load evolution and geometrical configuration (specimen central radius) up to macroscopic failure, with equivalent plastic strains reaching values as high as $\overline{\boldsymbol{\varepsilon}}_{p}=1.355$. Therefore, based on the fact that metal forming operations involve large plastic deformations, parameter identification using optimization-based strategies are highly recommended.

The present work investigated Modified Voce and Bergström hardening laws in association with an optimization-based strategy. In both cases, the technique required compliance of both load evolution and geometrical configuration (specimen central radius). The simulations indicated that Bergström equation provided smaller approximation errors than the Modified Voce hardening law. Therefore, Bergström equation is recommended to describe the yield stress evolution for the AISI 304 stainless steel.

\section{Acknowledgements}

The authors acknowledge the financial support provided by the Brazilian funding agency $\mathrm{CNPq}$ - (National Council for Scientific and Technological Development), Grant no. 303412/2016-0.

\section{References}

1. Kashyap BP, McTaggart K, Tangri K. Study on the substructure evolution and flow behaviour in type 316 stainless steel over the temperature range $21-900^{\circ} \mathrm{C}$. Philosophical Magazine A. 1988;57(1):97-114.

2. Kashyap BP, Tangri K. On the Hall-Petch relationship and substructural evolution in type $316 \mathrm{~L}$ stainless steel. Acta Metallurgica et Materialia. 1995;43(11):3971-3981.

3. Hollomon JH. Tensile deformation. Transactions of the Metallurgical Society of AIME. 1945;162:268-290.

4. Samuel KG, Rodriguez P. On power-law type relationships and the Ludwigson explanation for the stress-strain behaviour of AISI 316 stainless steel. Journal of Materials Science. 2005;40(21):5727-5731.

5. Samuel KG. Limitations of Hollomon and Ludwigson stressstrain relations in assessing the strain hardening parameters. Journal of Physics D: Applied Physics. 2006;39(1):203-212.

6. Ludwigson DC. Modified stress-strain relation for FCC metals and alloys. Metallurgical Transactions. 1971;2(10):28252828.
7. Samuel EI, Choudhary BK. Universal scaling of work hardening parameters in type $316 \mathrm{~L}(\mathrm{~N})$ stainless steel. Materials Science and Engineering: A. 2010;527(27-28):7457-7460.

8. Hertelé S, De Waele W, Denys R. A generic stress-strain model for metallic materials with two-stage strain hardening behaviour. International Journal of Non-Linear Mechanics. 2011;46(3):519-531.

9. Ramberg W, Osgood WR. Description of stress-strain curves by three parameters. Technical Note $N^{\circ}$. 902. Washington: National Advisory Committee for Aeronautics; 1943. 29 p.

10. El-Magd E. Modeling and Simulation of Mechanical Behavior In: Totten GE, Xie L, Funatani K, eds. Modeling and Simulation for Material Selection and Mechanical Design. New York: Marcel Dekker; 2004. p. 184-279.

11. Voce E. The relationship between stress and strain for homogeneous deformations. Journal of the Institute of Metals. 1948;74:537-562.

12. AshrafQJ, Prasad Reddy GV, Sandhya R, Laha K, Harmain GA. Simulation of low cycle fatigue stress-strain response in $316 \mathrm{LN}$ stainless steel using non-linear isotropic kinematic hardening model-A comparison of different approaches. Fatigue \& Fracture of Engineering Materials \& Structures. 2018;41(2):336-347.

13. Vaz Jr. M, Hulse ER, Tomiyama M. Identification of Inelastic Parameters of the AISI 304 Stainless Steel. In: Öchsner A, Altenbach H, eds. Engineering Design Applications II. Advanced Structured Materials. Cham: Springer; 2020. vol 113, p 17-35.

14. Vaz Jr. M, Cardoso EL, Muñoz-Rojas PA, Carniel TA, Luersen MA, Tomiyama M, et al. Identification of constitutive parameters - optimization strategies and applications. Materialwissenschaft und Werkstofftechnik. 2015;46(4-5):477-491.

15. De AK, Speer JG, Matlock DK, Murdock DC, Mataya MC, Comstock Jr. RJ. Deformation-induced phase transformation and strain hardening in type 304 austenitic stainless steel. Metallurgical and Materials Transactions A. 2006;37(6):1875-1886.

16. Swift HW. Plastic instability under plane stress. Journal of Mechanics and Physics of Solids. 1952;1(1):1-18.

17. Dimatteo A, Colla V, Lovicu G, Valentini R. Strain Hardening Behavior Prediction Model For Automotive High Strength Multiphase Steels. Steel Research International. 2015;86(12):1574-1582.

18. Viguier B. Dislocation densities and strain hardening rate in some intermetallic compounds. Materials Science and Engineering: A. 2003;349(1-2):132-135.

19. Mecking H, Kocks UF. Kinetics of flow and strain-hardening. Acta Metallurgica. 1981;29(11):1865-1875.

20. Panich S, Barlat F, Uthaisangsuk V, Suranuntchai S, Jirathearanat S. Experimental and theoretical formability analysis using strain and stress based forming limit diagram for advanced high strength steels. Materials \& Design. 2013;51:756-766.

21. Bergström Y.A dislocation model for the stress-strain behaviour of polycrystalline a-Fe with special emphasis on the variation of the densities of mobile and immobile dislocations. Materials Science and Engineering. 1970;5(4):193-200. 
22. Vaz Jr. M, Cardoso EL, Stahlschmidt J. Particle swarm optimization and identification of inelastic material parameters. Engineering Computations. 2013;30(7):936-960.

23. Nelder JA, Mead R. A Simplex Method for Function Minimization. The Computer Journal. 1965;7(4):308-313.

24. Lagarias JC, Reeds JA, Wright MH, Wright PE. Convergence properties of the Nelder-Mead simplex method in low dimensions. SIAM Journal of Optimization. 1998;9(1):112147.
25. Vaz Jr. M, Luersen MA, Muñoz-Rojas PA, Trentin RG. Identification of inelastic parameters based on deep drawing forming operations using a global-local hybrid Particle Swarm approach. Comptes Rendus Mécanique. 2016;344(4-5):319-334.

26. Associação Brasileira de Normas Técnicas (ABNT). NBR ISO 6892 - Metallic materials - tensile testing at ambient temperature (in Portuguese). Rio de Janeiro: ABNT; 2002.

27. Davis JR, ed. Tensile Testing. $2^{\text {nd }}$ ed. Materials Park: ASM International; 2004. 